\title{
Magnetic catalysis effect in the (2+1)-dimensional Gross-Neveu model with Zeeman interaction
}

\author{
K.G. Klimenko ${ }^{1,2}$, R.N. Zhokhov ${ }^{1}$ \\ ${ }^{1}$ Institute for High Energy Physics, 142281, Protvino, Moscow Region, Russia \\ 2 University "Dubna" (Protvino branch), 142281, Protvino, Moscow Region, Russia
}

\begin{abstract}
Magnetic catalysis of the chiral symmetry breaking and other magnetic properties of the (2+1)-dimensional Gross-Neveu model are studied taking into account the Zeeman interaction of spin-1/2 quasi-particles (electrons) with tilted (with respect to a system plane) external magnetic field $\vec{B}=\vec{B}_{\perp}+\vec{B}_{\|}$. The Zeeman interaction is proportional to magnetic moment $\mu_{B}$ of electrons. For simplicity, temperature and chemical potential are equal to zero throughout the paper. We compare in the framework of the model the above mentioned phenomena both at $\mu_{B}=0$ and $\mu_{B} \neq 0$. It is shown that at $\mu_{B} \neq 0$ the magnetic catalysis effect is drastically changed in comparison with the $\mu_{B}=0$ case. Namely, at $\mu_{B} \neq 0$ the chiral symmetry, being spontaneously broken by $\vec{B}$ at subcritical coupling constants, is always restored at $|\vec{B}| \rightarrow \infty$ (even at $\vec{B}_{\|}=0$ ). Moreover, it is proved in this case that chiral symmetry can be restored simply by tilting $\vec{B}$ to a system plane, and in the region $B_{\perp} \rightarrow 0$ the de Haas - van Alphen oscillations of the magnetization are observed. At supercritical values of coupling constant we have found two chirally non-invariant phases which respond differently on the action of $\vec{B}$. The first (at rather small values of $|\vec{B}|$ ) is a diamagnetic phase, in which there is an enhancement of chiral condensate, whereas the second is a paramagnetic chirally broken phase. Numerical estimates show that phase transitions described in the paper can be achieved at low enough laboratory magnetic fields.
\end{abstract}

\section{Introduction}

It is well known that during last three decades a lot of attention is paid to the investigation of (2+1)dimensional quantum field theories (QFT) under influence of different external conditions. In particular, the (2+1)-dimensional Gross-Neveu (GN) [1] type models are among the most popular [2-4]. There are several basic motivations for this interest. Since low dimensional theories have a rather simple structure, they can be used in order to develop our physical intuition for different physical phenomena taking place in real (3+1)-dimensional world (such as dynamical symmetry breaking [1-5], color superconductivity [6] etc). Another example of this kind is the spontaneous chiral symmetry breaking induced by external magnetic fields, i.e. the magnetic catalysis effect (see the recent reviews $[7,8]$ and references therein). For the first time this effect was also studied in terms of $(2+1)$ dimensional GN models [9]. In addition, low dimensional models are useful in elaborating new QFT methods like the large- $N$ technique $[1,3]$ and the optimized expansion method [10] etc. 
However, a more fundamental reason for the study of these theories is also well known. Indeed, there are a lot of condensed matter systems which, firstly, have a (quasi-)planar structure and, secondly, their low-energy excitation spectrum is described adequately by relativistic Dirac-like equation rather than by Schrödinger one.

\section{The model and its thermodynamic potential}

We suppose that some physical system is localized in the spatially two-dimensional plane perpendicular to the $\hat{z}$ coordinate axis of usual tree-dimensional space. Moreover, there is an external homogeneous and time independent magnetic field $\vec{B}$ tilted with respect to this plane. The corresponding (3+1)-dimensional vector potential $A_{\mu}$ is given by $A_{0,1}=0, A_{2}=B_{\perp} x, A_{3}=B_{\|} y$ We assume that the planar physical system consists of quasi-particles (electrons) with two spin projections, $\pm 1 / 2$, on the direction of magnetic field $\vec{B}$. Moreover, it is also supposed that their low-energy dynamics is described by the following (2+1)-dimensional Gross-Neveu type Lagrangian

$$
L=\sum_{k=1}^{2} \bar{\psi}_{k a}\left[\gamma^{0} i \partial_{t}+\gamma^{1} i \nabla_{1}+\gamma^{2} i \nabla_{2}-v(-1)^{k} \gamma^{0}\right] \psi_{k a}+\frac{G}{N}\left(\sum_{k=1}^{2} \bar{\psi}_{k a} \psi_{k a}\right)^{2},
$$

where $\nabla_{1,2}=\partial_{1,2}+i e A_{1,2}$ and the summation over the repeated index $a=1, \ldots, N$ of the internal $O(N)$ group is implied. For each fixed value of $k=1,2$ and $a=1, \ldots, N$ the quantity $\psi_{k a}(x)$ in (1) means the Dirac fermion field, transforming over a reducible 4-component spinor representation. We suppose that spinor fields $\psi_{1 a}(x)$ and $\psi_{2 a}(x)(a=1, \ldots, N)$ correspond to electrons with spin projections $1 / 2$ and $-1 / 2$ on the direction of an external magnetic field, respectively. In (1) the $v$-term is introduced in order to take into account the Zeeman interaction energy of electrons with external magnetic field $\vec{B}$. Hence, in our case $v=g_{S} \mu_{B}|\vec{B}| / 2$, where $|\vec{B}|=\sqrt{B_{\|}^{2}+B_{\perp}^{2}}, g_{S}$ is the spectroscopic Lande factor and $\mu_{B}$ is an electron magnetic moment, i.e. the Bohr magneton.

The model (1) is invariant under the discrete chiral transformation, $\psi_{k a} \rightarrow \gamma^{5} \psi_{k a}$. Certainly, there is the $O(N)$ invariance of the Lagrangian (1). Finally note that at $N=1$ the quasi-particle spectrum of the model (1) is just the same as in the monolayer graphene [19], but at $N>1$ one can interpret our results as occurring in the $N$-layered system.

In the following we use an auxiliary theory with the Lagrangian density

$$
\mathcal{L}=-\frac{N \sigma^{2}}{4 G}+\sum_{k=1}^{2} \bar{\psi}_{k a}\left(\gamma^{0} i \partial_{t}+\gamma^{1} i \nabla_{1}+\gamma^{2} i \nabla_{2}+\mu_{k} \gamma^{0}-\sigma\right) \psi_{k a},
$$

where $\mu_{1}=v, \mu_{2}=-v$ and from now on $v=\mu_{B}|\vec{B}|$ (in this formula and below the summation over repeated indices is implied). Clearly, the Lagrangians (1) and (2) are equivalent.

In the leading order of the large- $N$ approximation, the effective action $\mathcal{S}_{\text {eff }}(\sigma)$ of the considered model is expressed by means of the path integral over fermion fields

$$
\exp \left(i \mathcal{S}_{\mathrm{eff}}(\sigma)\right)=\int \prod_{k=1}^{2} \prod_{a=1}^{N}\left[d \bar{\psi}_{k a}\right]\left[d \psi_{k a}\right] \exp \left(i \int \mathcal{L} d^{3} x\right)
$$

In the leading order of the large- $N$ expansion the TDP is defined by the following expression:

$$
\int d^{3} x \Omega\left(M ; v, B_{\perp}\right)=-\left.\frac{1}{N} \mathcal{S}_{\mathrm{eff}}(\sigma(x))\right|_{\sigma(x)=M} .
$$




\subsection{The TDP in the general case $v \neq 0, B_{\perp} \neq 0$}

The TDP of the GN model with single $O(N)$ multiplet of Dirac spinors and at nonzero values of a chemical potential and $B_{\perp}$ was obtained, e.g., in [4, 21]. Taking into account the fact that in our case each of two $O(N)$ multiplets has its own chemical potential $\mu_{k}= \pm v$, one can easily generalize the results of $[4,21]$ and find the following expression for the renormalized TDP of the GN model (1):

$$
\Omega^{r e n}\left(M ; v, B_{\perp}\right)=\Omega^{r e n}\left(M ; B_{\perp}\right)-\frac{e B_{\perp}}{\pi} \sum_{n=0}^{\infty} s_{n} \theta\left(v-\varepsilon_{n}\right)\left(v-\varepsilon_{n}\right),
$$

where $s_{n}=2-\delta_{0 n}, \varepsilon_{n}=\sqrt{M^{2}+2 n e B_{\perp}}$, and the TDP $\Omega^{r e n}\left(M ; B_{\perp}\right)$

\section{Some properties of the model at $g>0$}

\subsection{Magnetic catalysis effect}

Suppose for a moment that $v$ does not depend on $|\vec{B}|$. There is a straight line $\lambda$ in the $\left(e g B_{\perp}, v\right)$-plane, tangent to a critical curve $v=v_{c}\left(B_{\perp}\right)$ at the point $B_{\perp}=0$, such that the whole $\left(e g B_{\perp}, v\right)$-region above $\lambda$ belongs to a symmetric phase of the model. It is clear that

$$
\lambda=\left\{\left(e g B_{\perp}, v\right): v=e g B_{\perp} / 2\right\} .
$$

Moreover, any straight line $v=\operatorname{keg} B_{\perp}$ with $k<1 / 2$ crosses the region of the $\left(e g B_{\perp}, v\right)$-plane, corresponding to a chiral symmetry broken phase.

The case $B_{\|}=0$, i.e. $B_{\perp}=|\vec{B}|$. Now, as it was intended from the very beginning, we suppose that $\vec{B}$ and $v$ are dependent quantities and, furthermore, that the external magnetic field $\vec{B}$ is perpendicular to a system plane, i.e. $B_{\perp}=|\vec{B}|$ and $v=\mu_{B} B_{\perp}$. Hence, in the case under consideration only the points of the straight line $v=\mu_{B} B_{\perp} \equiv \kappa e g B_{\perp}$ of the above mentioned $\left(e g B_{\perp}, v\right)$-plane are relevant to a real physical situation (evidently, $\kappa=\mu_{B} /(e g)$ ). So, if $\kappa>1 / 2$, i.e. at sufficiently small values of $g$, then the straight line $v=\mu_{B} B_{\perp}$ as a whole is above the line $\lambda$ (4), and spontaneous chiral symmetry breaking is forbidden in the system. However, if the coupling constant $g$ is greater than $g_{c}=2 \mu_{B} / e$, we have $\kappa<1 / 2$ and the line $v=\mu_{B} B_{\perp}$ is below $\lambda$. Obviously, in this case the straight line $v=\mu_{B} B_{\perp}$ crosses the region of the $\left(e g B_{\perp}, v\right)$-plane with chiral symmetry breaking. Hence, at $g>g_{c}$ chiral symmetry might be broken only for some finite interval of $B_{\perp}$-values. It means that the magnetic catalysis effect at $B_{\|}=0$ and $\mu_{B} \neq 0$, i.e. when the Zeeman interaction of electrons with magnetic field is taken into account, is qualitatively different from the case with $B_{\|}=0$ and $\mu_{B}=0$. Indeed, i) at $\mu_{B}=0$ the external (arbitrary small) magnetic field $B_{\perp}$ induces spontaneous chiral symmetry breaking at arbitrary values of $g>0$, whereas at $\mu_{B} \neq 0$ chiral symmetry might be broken by $B_{\perp}$ only at $g>g_{c}>0$. ii) If $g>g_{c}$, then at $\mu_{B} \neq 0$ the chiral symmetry is allowed to be spontaneously broken only for rather small values of $B_{\perp}$, i.e. at $B_{\perp}<B_{\perp c}$, where $0<B_{\perp c}<\infty$. The symmetry is restored at sufficiently high values of $B_{\perp}>B_{\perp c}$. In contrast, if the Zeeman interaction is neglected, we have $B_{\perp c}=\infty$ for arbitrary $g>0$.

To illustrate these circumstances we made some numerical investigations of the TDP (3) at $B_{\perp}=$ $|\vec{B}|$. For example, we have found that at $g=2.5 g_{c}, g=3.5 g_{c}$ and $g=5 g_{c}$ the corresponding critical values $B_{\perp c}$ of the perpendicular magnetic field at which there is a restoration of the chiral symmetry are the following, $e g^{2} B_{\perp c} \approx 0.059, e g^{2} B_{\perp c} \approx 0.518$ and $e g^{2} B_{\perp c} \approx 2.04$. Moreover, the behavior of the dynamical electron mass (or the gap) $M_{0}\left(B_{\perp}, v\right)$ vs $B_{\perp}$ in the particular case $g=5 g_{c}$ is presented in Fig. 1. It is clear from this figure that the gap is an increasing function vs $B_{\perp}$ up to a critical value $B_{\perp c}$, where it vanishes sharply, i.e. the first order phase transition occurs. 


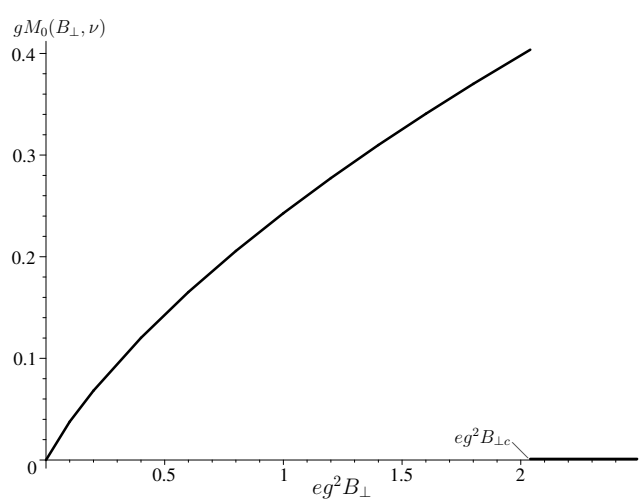

Figure 1. The case $g>0$ : The mass gap $M_{0}\left(B_{\perp}, v\right)$ vs $B_{\perp}$ in the particular case $B_{\|}=0$ and $g=5 g_{c} \equiv$ $10 \mu_{B} / e$. Here $e g^{2} B_{\perp c} \approx 2.04$.

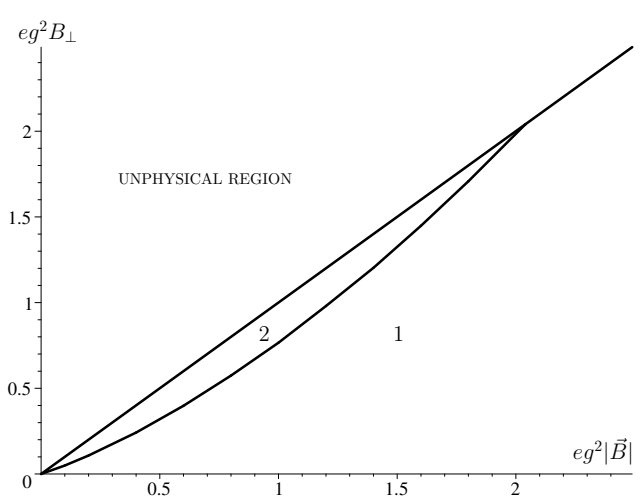

Figure 2. The case $g>0$ : The $\left(|\vec{B}|, B_{\perp}\right)$-phase portrait of the model at $g=5 g_{c} \equiv 10 \mu_{B} / e$. The numbers 1 and 2 denote the chirally symmetric and chirally broken phases, respectively. In the unphysical region of the figure $B_{\perp}>|\vec{B}|$. The boundary between 1 and 2 phases is the curve of the first order phase transitions.

The case $B_{\perp} \neq|\vec{B}|$. Now let us consider the general case when $B_{\|} \neq 0$, i.e. $B_{\perp} \neq|\vec{B}|$. In this case the mass gap $M_{0}\left(B_{\perp}, v\right)$ is really a function of two independent quantities, $B_{\perp}$ and $|\vec{B}|$, with an additional evident physical constraint $B_{\perp} \leq|\vec{B}|$. Investigating properties of the global minimum point of the TDP (3), depending on $B_{\perp}$ and $|\vec{B}|$, it is possible to obtain a corresponding phase portrait of the model. For a typical value of the parameter $g=5 g_{c}$ the phase structure of the model is presented in Fig. 2.

It is clear from the figure that at arbitrary small and perpendicular external magnetic field $\vec{B}$, such that $|\vec{B}|<B_{\perp c}$ (see the previous paragraphs), the system is in the chiral symmetry broken phase 2 . Then, the chiral symmetry can be restored by two qualitatively different ways. First, one may increase the strength of $\vec{B}$, or, second, it is possible simply to tilt $\vec{B}$ with respect to a system plane. In the last case, not too high deflection angle $\phi$ of the magnetic field is needed $\left(\phi \approx 45^{\circ}\right.$, where $\phi$ is the angle between $\vec{B}$ and the normal to the system plane) in order to restore the symmetry.

\subsection{Oscillations of the magnetization}

Now, let us consider the magnetization $m\left(|\vec{B}|, B_{\perp}\right)$ of the system under influence of an external tilted magnetic field at $g>0$. At fixed angle $\phi$ between $\vec{B}$ and the normal to the system plane, we define the magnetization by the following relation

$$
m\left(|\vec{B}|, B_{\perp}\right) \equiv-\left.\frac{d \Omega^{r e n}\left(M ; v, B_{\perp}\right)}{d|\vec{B}|}\right|_{M=M_{0}\left(B_{\perp}, v\right)},
$$

where $M_{0}\left(B_{\perp}, v\right)$ is the mass gap. It is possible to obtain

$$
m\left(|\vec{B}|, B_{\perp}\right)=-\left.\frac{B_{\perp}}{|\vec{B}|} \frac{\partial \Omega^{r e n}\left(M ; B_{\perp}\right)}{\partial B_{\perp}}\right|_{M=M_{0}\left(B_{\perp}, v\right)}+\left.\frac{e B_{\perp}}{\pi|\vec{B}|} \sum_{n=0}^{\infty} s_{n} \theta\left(v-\varepsilon_{n}\right)\left(2 v-\frac{\varepsilon_{n}^{2}+e n B_{\perp}}{\varepsilon_{n}}\right)\right|_{M=M_{0}\left(B_{\perp}, v\right)},
$$




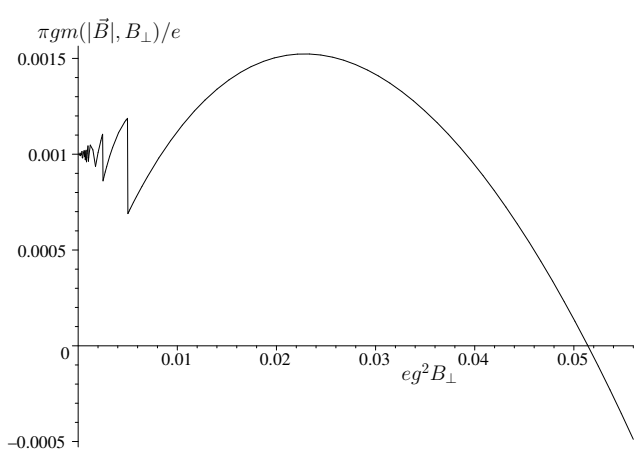

Figure 3. The case $g>0$ : Magnetization $m\left(|\vec{B}|, B_{\perp}\right)$ vs $B_{\perp}$ at fixed $e g^{2}|\vec{B}|=1$ and $g=5 g_{c} \equiv$ $10 \mu_{B} / e$.

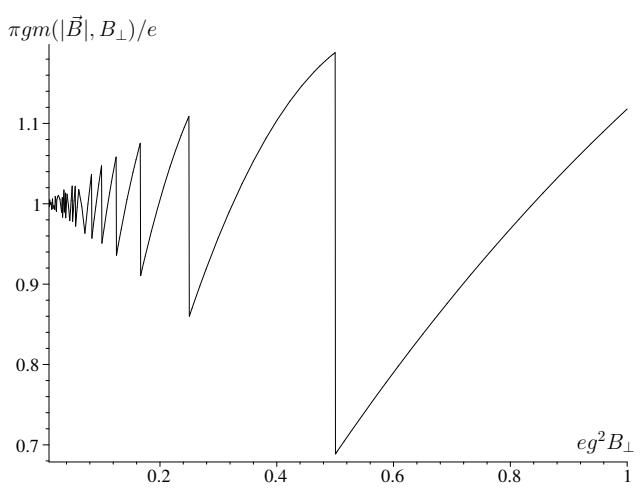

Figure 4. The case $g>0$ : Magnetization $m\left(|\vec{B}|, B_{\perp}\right)$ vs $B_{\perp}$ at fixed $e g^{2}|\vec{B}|=1$ and $g=$ $0.5 g_{c} \equiv \mu_{B} / e$.

where the notations of the expression (3) are used. The plot of the function (6) $m\left(|\vec{B}|, B_{\perp}\right)$ vs $B_{\perp}$ is presented in Figs 3 and 4 in two particular cases $g=5 g_{c}$ and $g=0.5 g_{c}$, correspondingly, at fixed value of $|\vec{B}|$ such that $e g^{2}|\vec{B}|=1$. It is clear from these figures that in the region of small values of $B_{\perp}$ the quantity (6) is a highly oscillating function.

Suppose that $|\vec{B}|$ is fixed. Since all terms of the series in (6) are positive quantities, one can conclude that in the region of sufficiently small $B_{\perp}$ magnetization as a whole are also positive quantities. Hence, at small values of $B_{\perp}$ the ground state of the model is a paramagnetic one. The situation can be changed, if $B_{\perp}$ approaches $|\vec{B}|$. In this case, depending on the relation between dimensionless parameters $e$ and $\mu_{B} / g$, one can obtain quite different magnetic properties of the ground state. Really, if $\mu_{B} / g \geq e$ (see, e.g., Fig. 4), then the magnetization is positive for all physical values of $B_{\perp}$, $0 \leq B_{\perp} \leq|\vec{B}|$, and the system is in the paramagnetic ground state. However, for a sufficiently small values of $\mu_{B} / g \ll e$ there is an interval of rather large values of $B_{\perp}$, the magnetization $m\left(|\vec{B}|, B_{\perp}\right)$ are negative quantities, so we have in this case a diamagnetic ground state of the system. For example, in Fig. 3 a graph of the magnetization $m\left(|\vec{B}|, B_{\perp}\right)$ vs $B_{\perp}$ is drown at fixed $|\vec{B}|$ and at $\mu_{B} / g=0.1 e$. Clearly, in this case the system is in the paramagnetic state if $e g^{2} B_{\perp}<0.051$, and it is a diamagnetic one at $e g^{2} B_{\perp}>0.051$.

It is possible to find the following asymptotic behavior of the magnetization (6) at $\vec{B}_{\perp} \rightarrow 0$ and arbitrary fixed $|\vec{B}|\left(\right.$ recall, $\left.v=\mu_{B}|\vec{B}|\right)$ :

$$
m\left(|\vec{B}|, B_{\perp}\right)=\frac{\mu_{B} v^{2}}{\pi}+\frac{\mu_{B} e B_{\perp}}{\pi^{2}} \sum_{n=1}^{\infty} \frac{1}{k} \sin \left(\frac{\pi k}{e B_{\perp}} v^{2}\right)+o\left(e B_{\perp}\right) .
$$

Remark, the leading asymptotic term in this expression, i.e. the first term in the right hand side of (7), is the magnetization corresponding to the TDP with zero $B_{\perp}$ component of an external magnetic field. Moreover, an infinite series in (7) is no more than Fourier expansion of the periodic function $f(x)$, where $x=v^{2} /\left(2 e B_{\perp}\right)$. Its period is equal to unity and in the interval $0<x<1$ it looks like $f(x)=\pi / 2-\pi x$.

Note, in condensed matter systems, both nonrelativistic [23, 24] and relativistic [25], magnetic oscillations usually occur in the presence of chemical potential $\mu$, i.e. in the systems with $\mu=0$ magnetic oscillations are absent as a rule. However, as it follows from our consideration in systems with 
planar structure magnetic oscillations can be induced even at $\mu=0$ by tilting the external magnetic field with respect to a system plane.

\section{Phase structure of the model at $g<0$}

In the present section we study the influence of an external magnetic field on the properties of the initial model (1) at $g<0$, i.e. at supercritical values of the bare coupling constant, $G>G_{c}$. Recall, when the Zeeman interaction is not taken into account the chiral symmetry breaking, induced originally in this case by a rather strong coupling, is enhanced additionally by external magnetic field (see, e.g., in $[9,13,15])$. It means that dynamical mass of electrons is an increasing function vs $B_{\perp}$ throughout the interval $0<B_{\perp}<\infty$ (in this case $B_{\|}$does not influence the properties of the model). It turns out that Zeeman interaction drastically changes properties of the model.

\subsection{The particular case, $|g|=\mu_{B} / e$.}

The case of perpendicular magnetic field. First, let us suppose that external magnetic field $\vec{B}$ is directed normally to a system plane, i.e. $B_{\perp}=|\vec{B}|$ and $B_{\|}=0$. For simplicity, we fix the value of $g$ by the relation $|g|=\mu_{B} / e$. Investigating in this case the TDP (3) as well as the gap equation, we have found the behavior of the mass gap $M_{0}\left(B_{\perp}, v\right)$ vs $B_{\perp}$ (it is the curve 1 in Fig. 5). It turns out that up to a some critical value $B_{\perp c_{1}}$ (such that $e g^{2} B_{\perp c_{1}} \approx 0.81$ ) the enhancement scenario is realized, i.e. the mass gap is an increasing function vs $B_{\perp}$. Moreover, in this chirally broken phase the gap $M_{0}\left(B_{\perp}, v\right)$ takes rather large values, such that $M_{0}\left(B_{\perp}, v\right)>v$. Consequently, the contribution to the magnetization $m\left(|\vec{B}|, B_{\perp}\right)$ coming from the Zeeman interaction vanishes, i.e. all terms of the series in (6) are zero. As a result, the magnetization in this phase is completely determined by an interaction of $\vec{B}$ with orbital angular momentum. Due to this reason $m\left(|\vec{B}|, B_{\perp}\right)$ is negative at $0<B_{\perp}<B_{\perp c_{1}}$ (see Fig. 5, where the curve 2 corresponds to a magnetization), and the ground state of this phase is a diamagnetic one.

Then, in the critical point $B_{\perp}=B_{\perp c_{1}}$ the mass gap $M_{0}\left(B_{\perp}, v\right)$ jumps to a significantly smaller nonzero value, and there is a phase transition of the first order to another chirally broken phase. Further increasing of $B_{\perp}$ leads to a restoration of the chiral symmetry at $B_{\perp}=B_{\perp c_{2}}$, where $e g^{2} B_{\perp c_{2}} \approx 0.94$. It is a second order phase transition, since in this point the mass gap $M_{0}\left(B_{\perp}, v\right)$ continuously turns into zero (see Fig. 5). Note also that both in the second chirally broken phase (at $B_{\perp c_{1}}<B_{\perp}<B_{\perp c_{2}}$ ) and in the chirally symmetric one (at $B_{\perp c_{2}}<B_{\perp}<\infty$ ) the magnetization of the system is positive, i.e. the ground states of these phases are paramagnetic (see Fig. 5).

The case of tilted magnetic field. Now, a few words about a response of the system with $g<0$ upon an arbitrarily directed external magnetic field, i.e. when $B_{\perp} \neq|\vec{B}|$. Numerical investigations of the TDP (3), where for simplicity we put $|g|=\mu_{B} / e$, bring us to the phase portrait of the model presented in Fig. 6. There the number 1 corresponds to a chirally symmetric paramagnetic phase, whereas notations 2 and 3 are used for two different chirally broken phases. The first of them, i.e. the phase 2 , is a diamagnetic with $m\left(|\vec{B}|, B_{\perp}\right)<0$, however the second one, i.e. the phase 3 , is a phase with paramagnetic ground state, since in this region $m\left(|\vec{B}|, B_{\perp}\right)>0$. Note, at $g<0$ one can also observe the oscillations of the magnetization only in the chirally symmetric phase 1 when $B_{\perp} \rightarrow 0$.

As it is clear from Figs 5 and 6 the presence of the Zeeman interaction significantly changes the behavior of the chiral symmetry under influence of an external both perpendicular and tilted magnetic field at $g<0$. Indeed, at $\mu_{B} \neq 0$ the enhancement of a chiral condensation in this case takes place only at sufficiently small values of $|\vec{B}|$, i.e. in the phase 2 of Fig. 6 (it means that fixing the tilting angle of the magnetic field we obtain the growth of the mass gap $M_{0}\left(B_{\perp}, v\right)$ at increasing $\left.|\vec{B}|\right)$. Further increasing of $|\vec{B}|$ leads ultimately to a chiral symmetry restoration. 


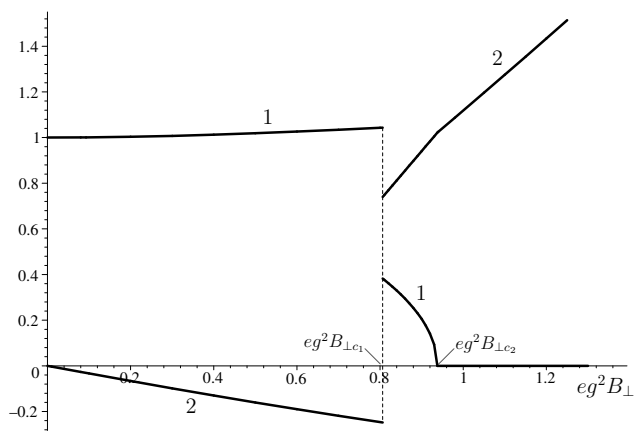

Figure 5. The case $g<0$ : Mass gap $M_{0}\left(B_{\perp}, v\right)$ and magnetization $m\left(|\vec{B}|, B_{\perp}\right)$ vs $B_{\perp}$ in the particular case $B_{\|}=0$ and $|g|=\mu_{B} / e$. Curves 1 and 2 are the plots of the dimensionless quantities $g M_{0}\left(B_{\perp}, v\right)$ and $\pi g m\left(|\vec{B}|, B_{\perp}\right) / e$, correspondingly. Here $e g^{2} B_{\perp c_{1}} \approx 0.81$ and $e g^{2} B_{\perp c_{2}} \approx 0.94$.

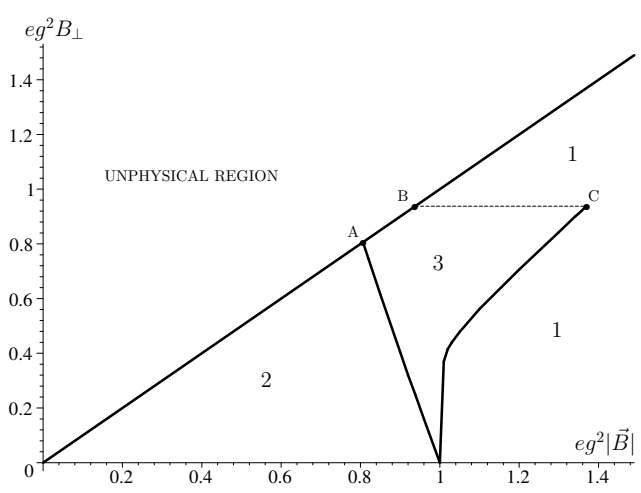

Figure 6. The case $g<0$ : The $\left(|\vec{B}|, B_{\perp}\right)$-phase portrait of the model at $|g|=\mu_{B} / e$. The numbers 1 denote the chirally symmetric phase, whereas the numbers 2 and 3 denote two different chirally broken phases (on the boundary between 2 and 3 the mass gap changes by a jump). The coordinates of the points $\mathrm{A}, \mathrm{B}$ and $\mathrm{C}$ approximately are $(0.81,0.81),(0.94,0.94)$ and $(1.37,0.94)$, correspondingly. The line $\mathrm{BC}$ is a curve of second order phase transitions; on the other lines the first order phase transitions take place.

\subsection{Phase structure in the general case}

Clearly, for other relations between $|g|$ and $\mu_{B}$, i.e. at $|g| \neq \mu_{B} / e$, the $\left(e g^{2}|\vec{B}|, e g^{2} B_{\perp}\right)$-phase portrait of the model might be quite different from Fig. 6. To imagine the phase structure of the model for an arbitrary, but fixed, relation between $|g|$ and $\mu_{B}$ it is very convenient to use for its description the new dimensionless parameters, $x=\mu_{B}|\vec{B}||g|$ and $y=e g^{2} B_{\perp}$ Assuming for a moment that $x$ and $y$ are fully independent quantities, it is possible to investigate the behavior of the global minimum point of the TDP (25) as a function of $x$ and $y$ and then to obtain the $(x, y)$-phase portrait of the model depicted in Figs 7 and/or 8. (The line L of these figures should be ignored in this case. Note also that in Fig. 8 the phase portrait is depicted for a more extended region of the parameter $y$.) There one can see only three different phases which were already presented in Fig. 6. So we use the same notations for them, 1, 2 and 3. In reality, there is a constraint between $x$ and $y$ which is due to the physical requirement $B_{\perp} \leq|\vec{B}|$. In terms of $x$ and $y$ it looks like $y \leq c x$, where $c=e|g| / \mu_{B}$, i.e. not the whole $(x, y)$-plates of Figs 7 and 8 can be considered as a phase diagram, but only those areas which are below the line $\mathrm{L}$. The points of the line $\mathrm{L}$ correspond to a perpendicular external magnetic field, i.e. we have $B_{\perp}=|\vec{B}|$ on the line L. Clearly, if the quantity $c=e|g| / \mu_{B}$ varies, then the line L of Figs 7 and 8 changes its slope and, as a result, the allowed physical region which is below $\mathrm{L}$ is also changed. However, the positions and forms of the critical curves in Figs 7, 8 are not changed at different values of the parameter $c$.

It is easily seen from Fig. 8 that inside the interval $3<y<11$ the critical curve $l$ of the phase diagram can be approximated by a straight line with a slope coefficient $c^{*} \approx 28$. Extrapolating this behavior of the curve $l$ to the region with higher $y$-values, one can conclude that a typical phase portrait 


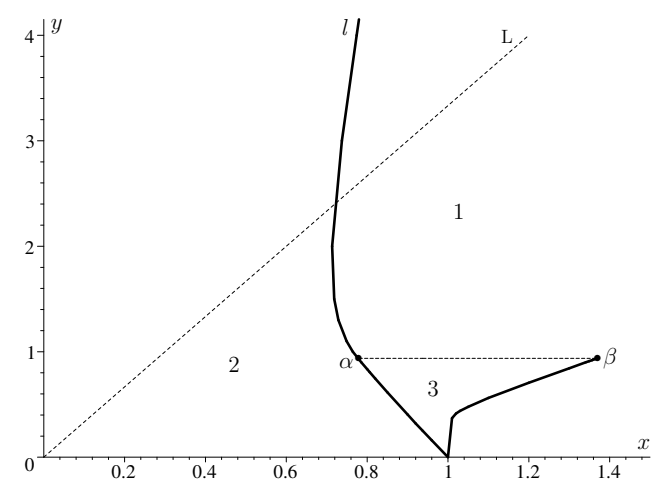

Figure 7. The case $g<0$ : The $(x, y)$-phase diagram of the model, where $x=\mu_{B}|\vec{B}||g|$ and $y=e g^{2} B_{\perp}$, typical for values of $c \equiv e|g| / \mu_{B}<$ $c^{*} \approx 28$. Physical region of the diagram corresponding to $B_{\perp} \leq|\vec{B}|$ relation lies just below the line $\mathrm{L}=\{(x, y): y=c x\}$. The notations 1,2 and 3 for different phases of the system are the same as in Fig. 6. First order phase transitions occur on the solid curves. On the line $\alpha \beta$ second order phase transitions take place. $\alpha \approx(0.71,0.94)$, $\beta \approx(1.37,0.94)$.

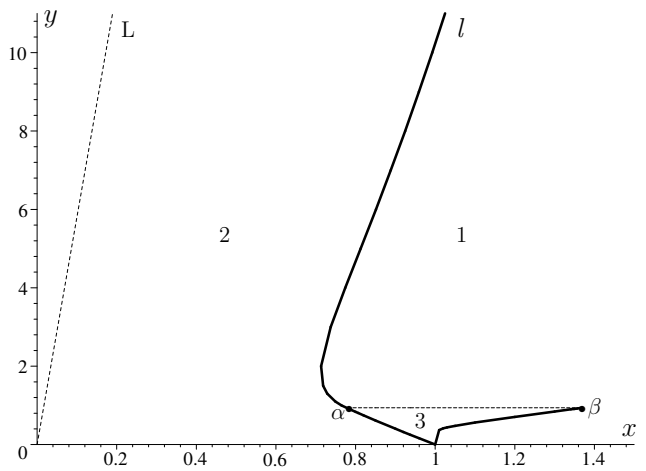

Figure 8. The case $g<0$ : The $(x, y)$-phase $\mathrm{di}$ agram of the model, where $x=\mu_{B}|\vec{B}||g|$ and $y=$ $e g^{2} B_{\perp}$, typical for values of $c \equiv e|g| / \mu_{B}>c^{*} \approx 28$. Physical region of the diagram, corresponding to $B_{\perp} \leq|\vec{B}|$ relation, lies just below and/or to the right of the line $\mathrm{L}=\{(x, y): y=c x\}$. Other notations are the same as in Fig. 7.

of the initial model corresponding to the weak coupling $|g|$, such that $c=e|g| / \mu_{B}<c^{*}$, is presented in Fig. 7 (it is the region just below the line L). In this case the line L certainly crosses critical curve $l$ of a phase portrait, i.e. it passes through several different phases, including the chirally symmetric phase 1 . As a result, one can see that at $c<c^{*}$ the chiral symmetry is always restored at $|\vec{B}| \rightarrow \infty$ irrespective of the magnetic field directions (even at a perpendicular magnetic field). In particular, the case $c=1$ was considered in details in the previous section IV A, and Fig. 7 at $c=1$ coincides with the phase diagram of Fig. 6.

In contrast, if $c>c^{*}$ then a typical phase portrait of the model is depicted in Fig. 8 (it is a region which is below and/or to the right of the line L). Clearly, in this case the line L does not cross any of the critical curves of the phase diagram, and at arbitrary values of a perpendicular magnetic field the chiral symmetry cannot be restored, since we move along the line L when $B_{\perp}=|\vec{B}|$ increases. However, if $|\vec{B}|$ reaches the values corresponding to $x>0.7$, then in this case at fixed $|\vec{B}|$ it is also possible to restore the symmetry by tilting the magnetic field away from the normal direction. In particular, if the parameter $x$ lies, e.g., in the interval $0.7<x<1.4$ (see Fig. 8), then a number of phase transitions can occur in the system that are also caused only by the inclination of an external magnetic field.

\subsection{Numerical estimates in the context of condensed matter physics}

Now let us estimate the order of magnitude of the magnetic field at which the phase transitions of Figs 6, 7, 8 might take place in (2+1)-dimensional condensed matter systems. To this end it is necessary to take into account in the Lagrangian (1) the Fermi velocity of quasi-particles $v_{F} \neq 1$. Using the 
same calculational technique as in Sec. II of the present paper and/or, e.g., in [18, 26], it is possible to obtain the thermodynamic potential $\Omega_{v_{F}}$ for the case $v_{F} \neq 1$. Indeed, there is a very simple connection between $\Omega_{v_{F}}$ and the renormalized TDP (3) corresponding to $v_{F}=1$. Namely, one should perform in (3) the replacements $e B_{\perp} \rightarrow e v_{F}^{2} B_{\perp}, g \rightarrow g / v_{F}$ (note, the Zeeman term $\mu_{B}|\vec{B}|$ remains unchanged in this case) and then multiply the obtained expression by the factor $1 / v_{F}^{2}$.

Suppose that $g<0$. Then, in the particular case of $\vec{B}=0$ the TDP $\Omega_{v_{F}}$ thus obtained from the TDP $V(M)$ of the case $v_{F}=1$ has already the global minimum at the point $M_{0 F} \equiv-v_{F} / g$ (it is the mass gap of the system). Since in all numerical calculations of the case $v_{F}=1$ an arbitrary dimensional quantity is converted into a dimensionless one by multiplying it with an appropriate powers of $|g|$, in the case $v_{F} \neq 1$ the powers of $|g| / v_{F}$ should be used instead. So, at $v_{F} \neq 1$ the analogs of the $(x, y)$-phase diagrams of Figs 7,8 are just the same figures, but with the new $x_{F^{-}}, y_{F}$-axes, where $x_{F}=x / v_{F} \equiv \mu_{B}|\vec{B} \| g| / v_{F}$, and $y_{F}=y$. (In the following, when referring to Figs 7, 8 in the case $v_{F} \neq 1$, we imply that instead of $x$ and $y$ the new parameters $x_{F}$ and $y_{F}$ should be used in these figures.) The line $\mathrm{L}$, below which the physical region is arranged, has the form $y_{F}=c_{F} x_{F}$, where $c_{F} \equiv c v_{F}=e|g| v_{F} / \mu_{B}=e v_{F}^{2} /\left(\mu_{B} M_{0 F}\right)$. It is clear from Figs 7,8 that at $B_{\perp}=0$ and $v_{F} \neq 1$ the phase transition of the first order occurs at in-plane magnetic field $\left|\vec{B}_{0}\right|$ corresponding to $x_{F}=1$, i.e. $\left|\vec{B}_{0}\right|=v_{F} /\left(|g| \mu_{B}\right)=M_{0 F} / \mu_{B}$. Since the value of the mass gap $M_{0 F}$ in condensed matter systems is typically of the order of 1-10 meV, one can easily obtain that the magnitude of the critical magnetic field $\left|\vec{B}_{0}\right|$ is of order of 14-140 Teslas, correspondingly. It is clear from Figs 7, 8 that at $B_{\perp} \neq 0$ the magnitudes of $|\vec{B}|$, at which one can observe phase transitions, are even less and might be as small as $0.7\left|\vec{B}_{0}\right|$.

If $v_{F}=1 / 300$ and $g_{S}=2$, as in graphene, then the slope factor $c_{F}$ of the line $\mathrm{L}$ is approximately equal to $10^{3}$ at $M_{0 F}=10 \mathrm{meV}$, whereas it is of order of $10^{4}$ at $M_{0 F}=1 \mathrm{meV}$, i.e. $c_{F} \gg c^{*} \approx 28$. Hence, just the phase diagram of Fig. 8 refers to graphene-like planar systems.

Note, up to now we have estimated phase transitions in the systems with $v_{F}=1 / 300$. However, still smaller values of the critical magnetic field $\left|\vec{B}_{0}\right|$ are realized in the planar gapless semiconductors at smaller values of $v_{F}$, e.g., at $v_{F}=1 / 3000$. In addition, in this case the slope factor $c_{F}$ of the line $\mathrm{L}$ might be extremely small, i.e. $c_{F} \sim 1$. So, just the phase diagram of Fig. 6 with a variety of phase transitions is relevant for such condensed matter systems.

In conclusion, we see that the effects which are due to the Zeeman interaction can be observed in real condensed matter systems at reasonable laboratory magnitudes of external magnetic fields.

\section{Summary and conclusions}

In the present paper we investigate (at zero temperature and chemical potential) the response of the $(2+1)$-dimensional GN model (1) upon the action of external magnetic field $\vec{B}$. The model describes a four-fermion self-interaction of quasi-particles (electrons) with spin 1/2. In addition, it describes the interaction of $\vec{B}$ both with orbital angular momentum of electrons and with their spin. The last is known as the Zeeman interaction, and it is proportional to electron magnetic moment $\mu_{B}$ which is a free model parameter in our consideration. So at $\mu_{B}=0$ the properties of the model were considered, e.g., in $[9,13,15]$, where in particular it was established that an external perpendicular magnetic field $\vec{B}_{\perp}$ induces spontaneous chiral symmetry breaking at $G<G_{c}$, or it enhances chiral condensation at $G>G_{c}$. (Such an ability of an external magnetic field is called the magnetic catalysis effect.) Moreover, in this case the system responds diamagnetically on the influence of external magnetic field, i.e. its magnetization is negative. In addition, there are no magnetic oscillations of any physical quantity if the Zeeman interaction is not taken into account. 
In the paper we study the modifications that appear both in the magnetic catalysis effect and in the magnetization phenomena of the system when Zeeman interaction is taken into consideration, i.e. at $\mu_{B} \neq 0$. To this end, we have obtained in the leading order of the large- $N$ expansion technique the renormalized thermodynamic potential $\Omega^{r e n}\left(M ; v, B_{\perp}\right)$ (3), where $v=\mu_{B}|\vec{B}|$. The behavior of the global minimum point of this quantity with respect to $M$ defines the phase structure of the model, whereas its derivative with respect to $|\vec{B}|$ gives us the magnetization. Note also that the renormalized TDP (3) depends no more on the bare coupling $G$. Instead, it appears the dependence of the TDP on the new finite parameter $g$ (Note that the values $g>0(g<0)$ correspond to the region $G<G_{c}$ $\left(G>G_{c}\right)$.) The main results of our investigations are the following.

i) We have found that at $\mu_{B} \neq 0$ and $g>0$ there is a critical coupling constant $g_{c}=2 \mu_{B} / e$ such that at $g>g_{c}$ an arbitrary rather weak external magnetic field $\vec{B}$ induces spontaneous chiral symmetry breaking provided that there is not too great a deviation of $\vec{B}$ from a vertical as well as that $|\vec{B}|<B_{c}(g)$, where $0<B_{c}(g)<\infty$ (see Fig. 2). At $0<g<g_{c}$ chiral symmetry cannot be broken by an external magnetic field. (In contrast, at $\mu_{B}=0$ and any values of $g>0$ the chiral symmetry breaking is induced by arbitrary external magnetic field $\vec{B}$ such that $\vec{B}_{\perp} \neq 0$.)

ii) Suppose that $\mu_{B} \neq 0, g>g_{c}>0$ and chiral symmetry is broken, i.e. $\vec{B}$ has a rather large $B_{\perp}$ component. Then chiral symmetry can be restored simply by tilting magnetic field to a system plane, i.e. without any increase of its modulus $|\vec{B}|$.

iii) We have shown that at $\mu_{B} \neq 0, g>0$ and arbitrary fixed $|\vec{B}| \neq 0$ one can observe oscillations of the magnetization in the region of small values of $B_{\perp}$ (see Figs 3 and 4).

iv) If $\mu_{B} \neq 0$ and $g<0$, then the phase structure and magnetic properties of the model are much richer than in the case of $\mu_{B}=0, g<0$. Indeed, it is clear from Figs 6, 7 and 8 that at non-vanishing Zeeman interaction the phase portrait of the model contains at least two chirally nonsymmetric phases, denoted as 2 and 3 . In the phase 2 , which is a diamagnetic one, the enhancement of the chiral symmetry is occurred, whereas in the paramagnetic phase 3 it is absent. Moreover, if in addition the parameter $c \equiv e|g| / \mu_{B}<c^{*} \approx 28$, then at sufficiently high values of $|\vec{B}|$ (even at a perpendicular magnetic field) the restoration of the chiral symmetry is occurred in the model. In contrast, at $\mu_{B}=0$ and $g<0$ only the diamagnetic phase 2 with enhancement of the chiral symmetry breaking is realized in the model at arbitrary values and directions of $\vec{B}$, such that $B_{\perp}>0$.

v) Assuming that the critical line $l$ of Fig. 8 can be extrapolated to the region $y \equiv e g^{2} B_{\perp}>11$ by a straight line with a slope coefficient $c^{*} \approx 28$, we see that at $g<0$ and $c \equiv e|g| / \mu_{B}>c^{*}$ the line L of Fig. 8 does not cross any of the critical curves of the figure. So, in this case at an arbitrary perpendicular magnetic field chiral symmetry cannot be restored. However, tilting the magnetic field away from a normal position, it is possible to restore the symmetry. As our numerical estimates show (see in Sec. IV C), just this situation is typical for graphene-like planar systems.

\section{References}

[1] D. J. Gross and A. Neveu, Phys. Rev. D 10, 3235 (1974).

[2] G. W. Semenoff and L. C. R. Wijewardhana, Phys. Rev. Lett. 63, 2633 (1989); Phys. Rev. D 45, 1342 (1992).

[3] B. Rosenstein, B. J. Warr and S. H. Park, Phys. Rep. 205, 59 (1991).

[4] A. S. Vshivtsev, B. V. Magnitsky, V. C. Zhukovsky and K. G. Klimenko, Phys. Part. Nucl. 29, 523 (1998) [Fiz. Elem. Chast. Atom. Yadra 29, 1259 (1998)].

[5] T. Appelquist and M. Schwetz, Phys. Lett. B 491, 367 (2000); S.J. Hands, J.B. Kogut and C.G. Strouthos, Phys. Rev. D 65, 114507 (2002). 
[6] D. Ebert, K. G. Klimenko and H. Toki, Phys. Rev. D 64, 014038 (2001); H. Kohyama, Phys. Rev. D 77, 045016 (2008); Phys. Rev. D 78, 014021 (2008).

[7] V. de la Incera, AIP Conf. Proc. 1361, 74 (2011) [arXiv:1004.4931].

[8] I. A. Shovkovy, arXiv:1207.5081 [hep-ph].

[9] K. G. Klimenko, Z. Phys. C 54, 323 (1992); Teor. Mat. Fiz. 89, 211 (1991) [Theor. Math. Phys. 89, 1161 (1991)]; Teor. Mat. Fiz. 90, 3 (1992) [Theor. Math. Phys. 90, 1 (1992)]; V. P. Gusynin, V. A. Miransky and I. A. Shovkovy, Phys. Rev. Lett. 73, 3499 (1994).

[10] K. G. Klimenko, Z. Phys. C 50, 477 (1991); Mod. Phys. Lett. A 9, 1767 (1994).

[11] A. S. Davydov, Phys. Rep. 190, 191 (1990); M. Rotter, M. Tegel and D. Johrendt, Phys. Rev. Lett. 101, 107006 (2008).

[12] A. H. Castro Neto, F. Guinea, N. M. R. Peres, K. S. Novoselov and A. K. Geim, Rev. Mod. Phys. 81, 109 (2009).

[13] G. W. Semenoff, I. A. Shovkovy and L. C. R. Wijewardhana, Mod. Phys. Lett. A 13, 1143 (1998).

[14] I. V. Fialkovsky and D. V. Vassilevich, arXiv:1111.3017 [hep-th].

[15] V. C. Zhukovsky, K. G. Klimenko, V. V. Khudyakov and D. Ebert, JETP Lett. 73, 121 (2001); V. C. Zhukovsky and K. G. Klimenko, Theor. Math. Phys. 134, 254 (2003); E. J. Ferrer, V. P. Gusynin and V. de la Incera, Mod. Phys. Lett. B 16, 107 (2002); Eur. Phys. J. B 33, 397 (2003).

[16] L. H. C. M. Nunes, R. L. S. Farias and E. C. Marino, Phys. Lett. A 376, 779 (2012).

[17] B. Roy and I. F. Herbut, Phys. Rev. B 83, 195422 (2011).

[18] H. Caldas and R. O. Ramos, Phys. Rev. B 80, 115428 (2009).

[19] V. P. Gusynin, V. A. Miransky, S. G. Sharapov, and I. A. Shovkovy, Phys. Rev. B 74, 195429 (2006); E. V. Gorbar, V. P. Gusynin, V. A. Miransky, and I. A. Shovkovy, Phys. Scr. T 146, 014018 (2012) [arXiv:1105.1360].

[20] E. J. Ferrer and V. de la Incera, Phys. Rev. Lett. 102, 050402 (2009); Nucl. Phys. B 824, 217 (2010).

[21] A. S. Vshivtsev, B. V. Magnitsky and K. G. Klimenko, JETP Lett. 62, 283 (1995); Theor. Math. Phys. 106, 319 (1996).

[22] N. V. Gubina, K. G. Klimenko, S. G. Kurbanov and V. C. Zhukovsky, Phys. Rev. D 86, 085011 (2012).

[23] W. J. de Haas and P. M. van Alphen, Proc. Amsterdam Acad. 33, 1106 (1936); D. Shoenberg, Magnetic oscillations in metals, Cambridge Univ. Press, Cambridge, 1984.

[24] I. M. Lifshitz, Selected Works. Electronic Theory of Metals, Physics of Polymers and Bipolymers [in Russian], Nauka, Moscow, 1994; E. M. Lifshitz and L. P. Pitaevski, Statistical Physics, Pergamon, Oxford, 1980.

[25] A. S. Vshivtsev and K. G. Klimenko, J. Exp. Theor. Phys. 82, 514 (1996) [Zh. Eksp. Teor. Fiz. 109, 954 (1996)]; D. Ebert, K. G. Klimenko, M. A. Vdovichenko and A. S. Vshivtsev, Phys. Rev. D 61, 025005 (2000).

[26] R. O. Ramos and P. H. A. Manso, Phys. Rev. D 87, 125014 (2013) [arXiv:1303.5463 [hep-ph]]. 